Electronic Supporting information

for

\title{
Impact of the Copper-Induced Local Framework Deformation on the Mechanism of Structural Phase Transition in $\left[\left(\mathrm{CH}_{3}\right)_{2} \mathrm{NH}_{2}\right]\left[\mathrm{Zn}(\mathrm{HCOO})_{3}\right]$ Hybrid Metal-Formate Perovskite
}

Paulina Peksa ${ }^{1}$, Justyna Trzmiel ${ }^{1}$, Katarzyna Fedoruk ${ }^{1}$, Anna Gągor ${ }^{2}$, Mantas Šimènas ${ }^{3}$, Aneta Ciupa $^{2}$, Sebastian Pawlus ${ }^{4}$, Juras Banys ${ }^{3}$, Mirosław Mączka ${ }^{2}$ and Adam Sieradzki ${ }^{*}$

1. Faculty of Fundamental Problems of Technology, Wrocław University of Science and Technology, WybrzeżeWyspiańskiego 27, 50-370 Wrocław, Poland

2. Institute of Low Temperature and Structure Research, Polish Academy of Sciences, Box 1410, 50-950 Wrocław 2, Poland

3. Faculty of Physics, Vilnius University, Sauletekio 9, LT-10222 Vilnius, Lithuania

4. Institute of Physics, University of Silesia, ul. 75 PulkuPiechoty 1, 41-500 Chorzów, Poland

Email: adam.sieradzki@pwr.edu.pl

Table S1 The $\mathrm{Zn}$ and $\mathrm{Cu}$ content determined by the ICP-OES analysis.

\begin{tabular}{ccc}
\hline Sample & $\mathbf{Z n ~ ( m o l \% )}$ & Cu (mol\%) \\
\hline DMAZn $_{0.999} \mathrm{Cu}_{0.001}$ & 99.7 & 0.2 \\
$\operatorname{DMAZn}_{0.995} \mathrm{Cu}_{0.005}$ & 99.3 & 0.6 \\
$\mathrm{DMAZn}_{0.99} \mathrm{Cu}_{0.01}$ & 98.8 & 1.3 \\
$\mathrm{DMAZn}_{0.98} \mathrm{Cu}_{0.02}$ & 98.1 & 2.1 \\
$\mathrm{DMAZn}_{0.97} \mathrm{Cu}_{0.03}$ & 96.7 & 3.5 \\
$\mathrm{DMAZn}_{0.95} \mathrm{Cu}_{0.05}$ & 94.3 & 5.4 \\
\hline
\end{tabular}


Table S2 Single-crystal x-ray diffraction experimental details

For all structures: system trigonal, $R-3 c: H, Z=3$. Experiments were carried out at $165 \mathrm{~K}$ with Mo $K a$ radiation using aXcalibur, Atlas. Absorption was corrected by multi-scan methods, CrysAlis PRO 1.171.38.43 (Rigaku Oxford Diffraction, 2015). Empirical absorption correction using spherical harmonics was implemented in SCALE3 ABSPACK scaling algorithm. H-atom parameters were constrained.

\begin{tabular}{|c|c|c|}
\hline & DMAZn_165K & DMAZn:Cu0.05_165K \\
\hline \multicolumn{3}{|l|}{ Crystal data } \\
\hline $\begin{array}{l}\text { Chemical formula } \\
M_{\mathrm{r}} \\
a, c(\AA) \\
V\left(\AA^{3}\right) \\
\mu\left(\mathrm{mm}^{-1}\right) \\
\text { Crystal size }(\mathrm{mm})\end{array}$ & $\begin{array}{l}\mathrm{C}_{10} \mathrm{H}_{22} \mathrm{~N}_{2} \mathrm{O}_{12} \mathrm{Zn}_{2} \\
493.03 \\
8.1837(13), \quad 22.110(5) \\
1282.4(5) \\
2.87 \\
0.20 \times 0.16 \times 0.13\end{array}$ & $\begin{array}{l}\mathrm{C}_{10} \mathrm{H}_{22} \mathrm{Cu}_{0.10} \mathrm{~N}_{2} \mathrm{O}_{12} \mathrm{Zn}_{1.90} \\
492.85 \\
8.1777(13), \quad 22.070(5) \\
1278.2(5) \\
2.87 \\
0.18 \times 0.16 \times 0.11\end{array}$ \\
\hline \multicolumn{3}{|l|}{ Data collection } \\
\hline $\begin{array}{l}\text { Radiation source } \\
T_{\min }, T_{\max } \\
\text { No. of measured, } \\
\text { independent and } \\
\text { observed }[I>2 \sigma(I)] \\
\text { reflections }\end{array}$ & $\begin{array}{l}\text { fine-focus sealed X-ray tube, Enhance } \\
\text { (Mo) X-ray Source } \\
0.652,1.000 \\
2568,277,272\end{array}$ & $\begin{array}{l}\text { fine-focus sealed X-ray tube, Enhance (Mo) } \\
\text { X-ray Source } \\
0.792,1.000 \\
5083,276,272\end{array}$ \\
\hline $\begin{array}{l}R_{\text {int }} \\
(\sin \theta / \lambda)_{\max }\left(\AA^{-1}\right)\end{array}$ & $\begin{array}{l}0.021 \\
0.610\end{array}$ & $\begin{array}{l}0.021 \\
0.609\end{array}$ \\
\hline \multicolumn{3}{|l|}{ Refinement } \\
\hline $\begin{array}{l}R\left[F^{2}>2 \sigma\left(F^{2}\right)\right], w R\left(F^{2}\right), S \\
\text { No. of reflections } \\
\text { No. of parameters } \\
(\Delta / \sigma)_{\max } \\
\left.\Delta\rangle_{\max }, \Delta\right\rangle_{\min }\left(\mathrm{e} \AA^{-3}\right)\end{array}$ & 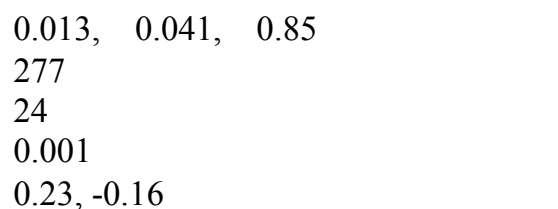 & $\begin{array}{l}0.012, \quad 0.039, \quad 0.81 \\
276 \\
23 \\
\& 1 t ; 0.001 \\
0.18,-0.17\end{array}$ \\
\hline
\end{tabular}

Computer programs: CrysAlis PRO 1.171.38.43 (Rigaku OD, 2015), SHELXL2018/3 (Sheldrick, 2018). 
Table S3. Selected geometric parameters $\left(\AA,{ }^{\circ}\right)$

\begin{tabular}{|c|c|c|c|}
\hline \multicolumn{4}{|l|}{$D M A Z n \_165 K$} \\
\hline $\mathrm{Zn}-\mathrm{O} 1^{\mathrm{i}}$ & $2.1088(8)$ & $\mathrm{Zn}-\mathrm{O} 1^{\text {iv }}$ & $2.1088(8)$ \\
\hline $\mathrm{Zn}-\mathrm{O} 1^{\mathrm{ii}}$ & $2.1088(8)$ & $\mathrm{Zn}-\mathrm{O}^{\mathrm{v}}$ & $2.1088(8)$ \\
\hline $\mathrm{Zn}-\mathrm{O} 1$ & $2.1088(8)$ & $\mathrm{O} 1-\mathrm{C} 1$ & $1.2483(12)$ \\
\hline $\mathrm{Zn}-\mathrm{O} 1^{\mathrm{iii}}$ & $2.1088(8)$ & $\mathrm{C} 2-\mathrm{N} 1$ & $1.420(4)$ \\
\hline \multicolumn{4}{|c|}{ DMAZnCu0.05_165K } \\
\hline $\mathrm{Zn}-\mathrm{O} 1^{\mathrm{i}}$ & $2.1056(8)$ & $\mathrm{Zn}-\mathrm{O} 1^{\mathrm{iv}}$ & $2.1056(8)$ \\
\hline $\mathrm{Zn}-\mathrm{O} 1^{\mathrm{ii}}$ & $2.1056(8)$ & $\mathrm{Zn}-\mathrm{O}^{\mathrm{v}}$ & $2.1056(8)$ \\
\hline $\mathrm{Zn}-\mathrm{O} 1$ & $2.1056(8)$ & $\mathrm{O} 1-\mathrm{C} 1$ & $1.2472(11)$ \\
\hline $\mathrm{Zn}-\mathrm{O} 1^{\mathrm{iii}}$ & $2.1056(8)$ & $\mathrm{C} 2-\mathrm{N} 1$ & $1.414(3)$ \\
\hline \multicolumn{4}{|l|}{$D M A Z n \_165 K$} \\
\hline $\mathrm{O} 1^{\mathrm{i}}-\mathrm{Zn}-\mathrm{O} 1^{\mathrm{ii}}$ & $180.00(6)$ & 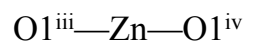 & $88.68(3)$ \\
\hline 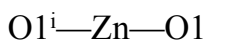 & $88.68(3)$ & $\mathrm{O} 1^{\mathrm{i}}-\mathrm{Zn}-\mathrm{O} 1^{\mathrm{v}}$ & $91.32(3)$ \\
\hline 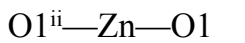 & $91.32(3)$ & $\mathrm{O} 1^{\mathrm{ii}-}-\mathrm{Zn}-\mathrm{O} 1^{\mathrm{v}}$ & $88.68(3)$ \\
\hline 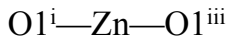 & $91.32(3)$ & $\mathrm{O} 1-\mathrm{Zn}-\mathrm{O} 1^{\mathrm{v}}$ & $88.68(3)$ \\
\hline 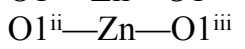 & $88.68(3)$ & 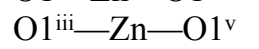 & $91.32(3)$ \\
\hline $\mathrm{O} 1-\mathrm{Zn}-\mathrm{O} 1^{\mathrm{iii}}$ & 180.0 & $\mathrm{O} 1^{\mathrm{iv}}-\mathrm{Zn}-\mathrm{O}^{\mathrm{v}}$ & $180.00(5)$ \\
\hline $\mathrm{O} 1^{\mathrm{i}-} \mathrm{Zn}-\mathrm{O} 1^{\mathrm{iv}}$ & $88.68(3)$ & $\mathrm{C} 1-\mathrm{O} 1-\mathrm{Zn}$ & $126.09(9)$ \\
\hline $\mathrm{O} 1^{\mathrm{ii}-} \mathrm{Zn}-\mathrm{O} 1^{\mathrm{iv}}$ & $91.32(3)$ & 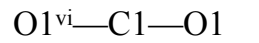 & 125.09 (17) \\
\hline $\mathrm{O} 1-\mathrm{Zn}-\mathrm{O} 1^{\text {iv }}$ & $91.32(3)$ & $\mathrm{C} 2-\mathrm{N} 1-\mathrm{C} 2^{\mathrm{vii}}$ & $119.4(4)$ \\
\hline \multicolumn{4}{|c|}{ DMAZnCu0.05_165K } \\
\hline $\mathrm{O} 1^{\mathrm{i}-}-\mathrm{Zn}-\mathrm{O} 1^{\mathrm{ii}}$ & $180.00(5)$ & 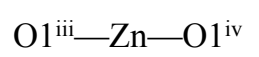 & $88.66(3)$ \\
\hline 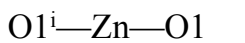 & $88.66(3)$ & $\mathrm{O} 1^{\mathrm{i}}-\mathrm{Zn}-\mathrm{O} 1^{\mathrm{v}}$ & $91.34(3)$ \\
\hline 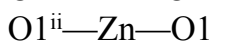 & $91.34(3)$ & 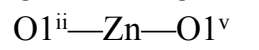 & $88.66(3)$ \\
\hline $\mathrm{O} 1^{\mathrm{i}-}-\mathrm{Zn}-\mathrm{O} 1^{\mathrm{iii}}$ & $91.34(3)$ & $\mathrm{O} 1-\mathrm{Zn}-\mathrm{O}^{\mathrm{v}}$ & $88.66(3)$ \\
\hline 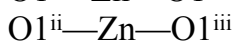 & $88.66(3)$ & 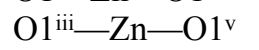 & $91.34(3)$ \\
\hline $\mathrm{O} 1-\mathrm{Zn}-\mathrm{O} 1^{\mathrm{iii}}$ & 180.0 & $\mathrm{O} 1^{\mathrm{iv}}-\mathrm{Zn}-\mathrm{O} 1^{\mathrm{v}}$ & $180.00(5)$ \\
\hline $\mathrm{O} 1^{\mathrm{i}-} \mathrm{Zn}-\mathrm{O} 1^{\mathrm{iv}}$ & $88.66(3)$ & $\mathrm{C} 1-\mathrm{O} 1-\mathrm{Zn}$ & $126.11(9)$ \\
\hline 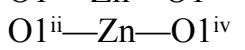 & $91.34(3)$ & $\mathrm{O} 1^{\mathrm{vi}-\mathrm{C} 1-\mathrm{O} 1}$ & $125.06(16)$ \\
\hline $\mathrm{O} 1-\mathrm{Zn}-\mathrm{O}^{\mathrm{iv}}$ & $91.34(3)$ & $\mathrm{C} 2-\mathrm{N} 1-\mathrm{C} 2^{\mathrm{vii}}$ & $119.9(4)$ \\
\hline
\end{tabular}

Table S4. Selected hydrogen-bond parameters

\begin{tabular}{|c|c|c|c|c|}
\hline$D-\mathrm{H} \cdots A$ & $D-\mathrm{H}(\AA) \mathrm{I}$ & $\mathrm{H} \cdots A(\AA)$ & $D \cdots A(\AA)$ & $D-\mathrm{H}^{\cdots} \cdots A\left(^{\circ}\right)$ \\
\hline \multicolumn{5}{|l|}{$D M A Z n \_165 K$} \\
\hline $\mathrm{N} 1-\mathrm{H} 1 \mathrm{~A} \cdots \mathrm{O} 1^{\mathrm{i}}$ & 0.96 & 2.59 & $3.2470(10)$ & 126.1 \\
\hline $\mathrm{N} 1-\mathrm{H} 1 \mathrm{~B} \cdots \mathrm{O} 1^{\mathrm{ii}}$ & 0.96 & 2.59 & $3.2470(10)$ & 126.0 \\
\hline $\mathrm{N} 1-\mathrm{H} 1 \mathrm{~A} \cdots \mathrm{O} 1^{\mathrm{iii}}$ & 0.96 & 1.97 & $2.885(3)$ & 159.4 \\
\hline $\mathrm{N} 1-\mathrm{H} 1 \mathrm{~B} \cdots \mathrm{O} 1^{\text {iv }}$ & 0.96 & 1.97 & $2.885(3)$ & 159.4 \\
\hline \multicolumn{5}{|c|}{ DMAZnCU005_165K } \\
\hline $\mathrm{N} 1-\mathrm{H} 1 \mathrm{~A} \cdots \mathrm{O} 1^{\mathrm{i}}$ & 0.96 & 2.59 & $3.2463(9)$ & 125.8 \\
\hline $\mathrm{N} 1-\mathrm{H} 1 \mathrm{~B} \cdots \mathrm{O} 1^{\mathrm{ii}}$ & 0.96 & 2.58 & $3.2463(9)$ & 126.1 \\
\hline $\mathrm{N} 1-\mathrm{H} 1 \mathrm{~A} \cdots \mathrm{O} 1^{\mathrm{iii}}$ & 0.96 & 1.97 & $2.889(3)$ & 159.4 \\
\hline $\mathrm{N} 1-\mathrm{H} 1 \mathrm{~B} \cdots \mathrm{O} 1^{\mathrm{iv}}$ & 0.96 & 1.97 & $2.889(3)$ & 159.5 \\
\hline
\end{tabular}

Symmetry code(s): (i) $x-y-1 / 3, x+1 / 3,-z+1 / 3$; (ii) $x+1 / 3, x-y-1 / 3, z+1 / 6$; (iii) $-y-2 / 3,-x-1 / 3, z+1 / 6$; (iv) $-x-1 / 3,-y-2 / 3$, $-z+1 / 3$. 


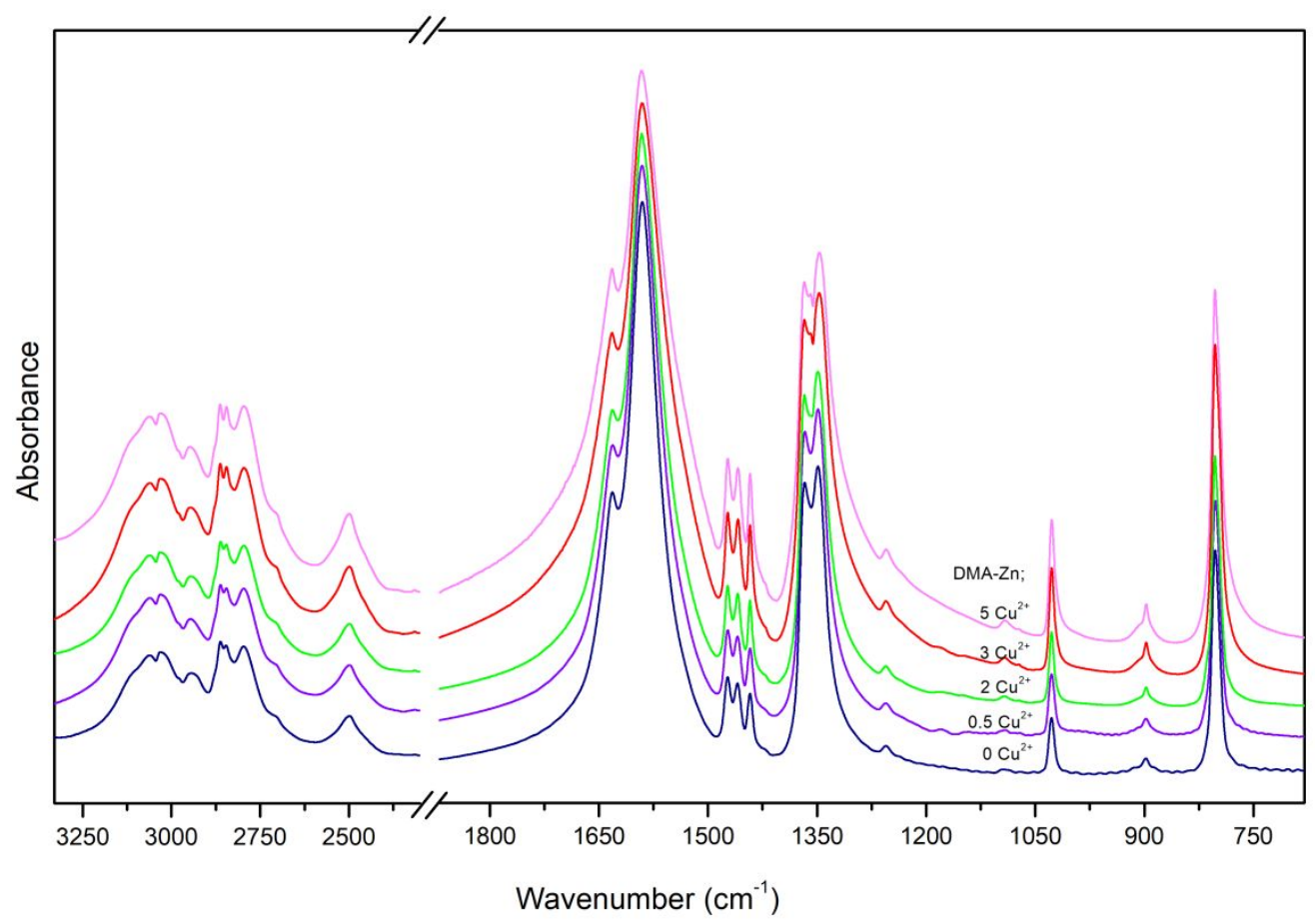

Figure S1. Mid-IR spectra of pure and Cu-doped DMAZn measured at room-temperature.

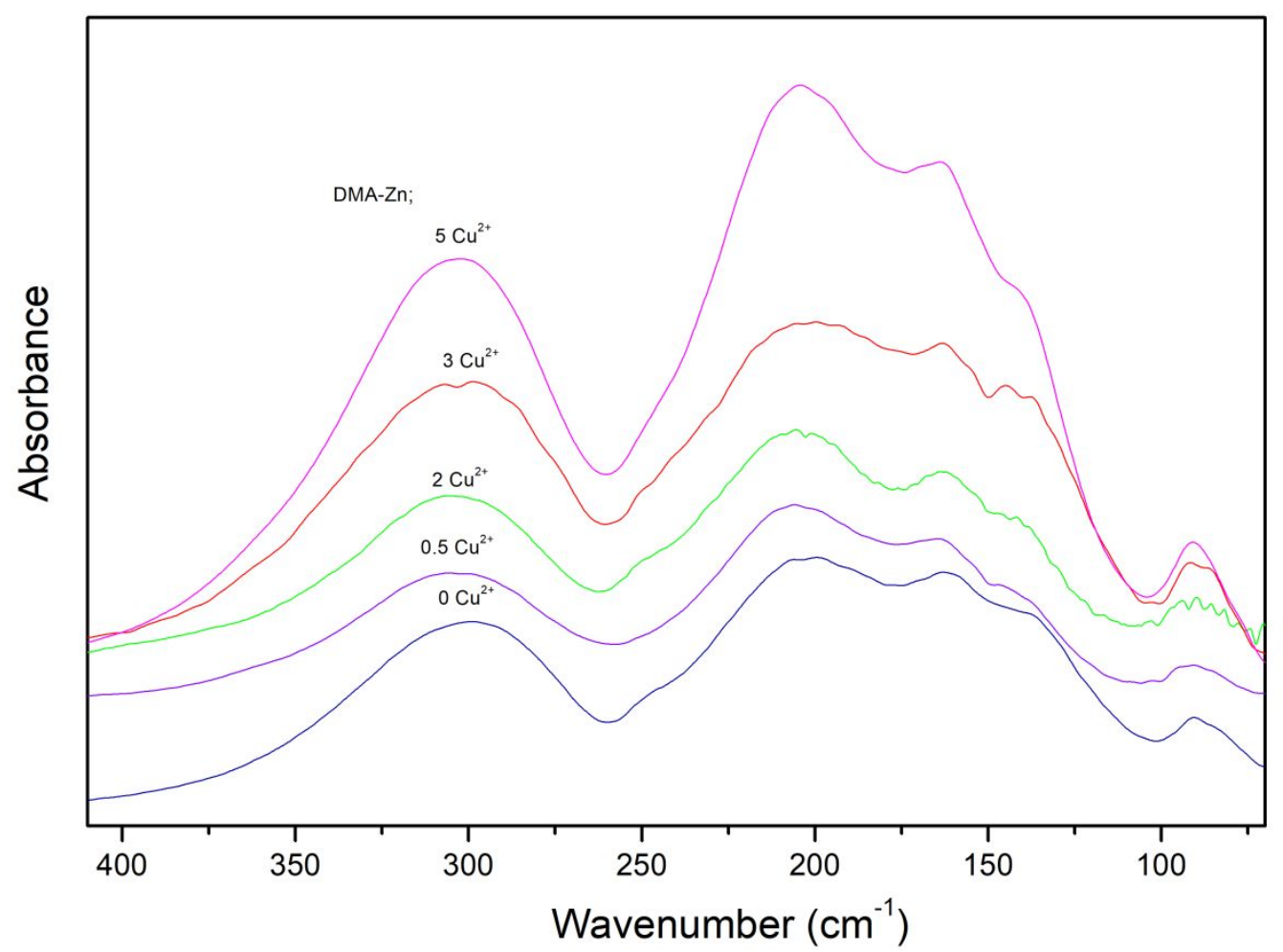

Figure S2. FAR-IR spectra of pure and $\mathrm{Cu}$-doped DMAZn measured at room-temperature. 
Table S5 Wavenumbers observed in the polycrystalline IR spectra of pure and Cu-doped DMAZn samples together with assignment proposed.

\begin{tabular}{|c|c|c|}
\hline DMAZn & $\mathrm{DMAZn}_{0.95} \mathrm{Cu}_{0.05}$ & Assignment \\
\hline $3066 w$ & $3066 w$ & $v\left(\mathrm{NH}_{2}\right)$ \\
\hline $3033 w$ & $3033 w$ & $v\left(\mathrm{NH}_{2}\right)$ \\
\hline $2949 w$ & $2949 w$ & $v\left(\mathrm{CH}_{3}\right)$ \\
\hline $2882 \mathrm{sh}$ & $2882 \mathrm{sh}$ & $v_{4}\left(\mathrm{HCOO}^{-}\right)+v_{5}\left(\mathrm{HCOO}^{-}\right)$ \\
\hline $2863 \mathrm{~m}$ & $2862 \mathrm{~m}$ & $v_{4}\left(\mathrm{HCOO}^{-}\right)+v_{5}\left(\mathrm{HCOO}^{-}\right)$ \\
\hline $2844 \mathrm{~m}$ & $2843 \mathrm{~m}$ & $v_{1}\left(\mathrm{HCOO}^{-}\right)$ \\
\hline $2798 \mathrm{~m}$ & $2798 \mathrm{~m}$ & $v\left(\mathrm{NH}_{2}\right)$ \\
\hline $2703 \mathrm{sh}$ & $2793 \mathrm{sh}$ & $2 v_{2}\left(\mathrm{HCOO}^{-}\right)$ \\
\hline $2502 \mathrm{~m}$ & $2502 \mathrm{~m}$ & $\rho\left(\mathrm{NH}_{2}\right)+\delta\left(\mathrm{NH}_{2}\right)$ \\
\hline $1632 \mathrm{~m}$ & $1632 \mathrm{~m}$ & $\delta\left(\mathrm{NH}_{2}\right)$ \\
\hline $1593 \mathrm{vs}$ & $1592 \mathrm{vs}$ & $v_{4}\left(\mathrm{HCOO}^{-}\right)$ \\
\hline $1473 w$ & $1473 w$ & $\delta_{\text {as }}\left(\mathrm{CH}_{3}\right)$ \\
\hline $1461 w$ & $1461 w$ & $\delta_{\mathrm{as}}\left(\mathrm{CH}_{3}\right)$ \\
\hline $1443 w$ & $1443 w$ & $\delta_{\mathrm{as}}\left(\mathrm{CH}_{3}\right)$ \\
\hline $1368 \mathrm{~s}$ & $1367 \mathrm{~s}$ & $v_{5}\left(\mathrm{HCOO}^{-}\right)$ \\
\hline $1360 \mathrm{~m}$ & $1359 \mathrm{~m}$ & $v_{5}\left(\mathrm{HCOO}^{-}\right)$ \\
\hline $1349 \mathrm{~m}$ & $1347 \mathrm{~m}$ & $v_{2}\left(\mathrm{HCOO}^{-}\right)$ \\
\hline $1256 w$ & $1256 \mathrm{w}$ & $\rho\left(\mathrm{CH}_{3}\right)$ \\
\hline $1094 \mathrm{vw}$ & $1095 \mathrm{vw}$ & $\rho\left(\mathrm{CH}_{3}\right)$ \\
\hline \multirow[t]{2}{*}{$1028 w$} & $1027 \mathrm{w}$ & $v_{\mathrm{as}}(\mathrm{CNC})$ \\
\hline & $910 w, b$ & $\rho\left(\mathrm{NH}_{2}\right)$ \\
\hline $899 w$ & $899 w$ & $v_{\mathrm{s}}(\mathrm{CNC})$ \\
\hline $803 \mathrm{~s}$ & $801 \mathrm{~s}$ & $v_{3}\left(\mathrm{HCOO}^{-}\right)$ \\
\hline $298 \mathrm{~s}$ & $302 \mathrm{~s}$ & $\mathrm{~T}^{\prime}\left(\mathrm{M}^{2+}\right)$ \\
\hline $246 \mathrm{sh}$ & $243 \mathrm{sh}$ & $\mathrm{T}^{\prime}\left(\mathrm{M}^{2+}\right)$ \\
\hline $202 \mathrm{~s}$ & $205 s$ & $\mathrm{~T}^{\prime}\left(\mathrm{HCOO}^{-}\right)$ \\
\hline $162 \mathrm{~m}$ & $163 \mathrm{~m}$ & L(DMA) \\
\hline $136 \mathrm{~m}$ & $142 \mathrm{~m}$ & $\mathrm{~L}\left(\mathrm{HCOO}^{-}\right)$ \\
\hline $85 \mathrm{w}$ & $91 \mathrm{w}$ & $\mathrm{T}^{\prime}(\mathrm{DMA})$ \\
\hline
\end{tabular}

Key: $v$ - stretching, $\delta$ - bending, $\rho$ - rocking vibration, L - librations, T' - translations; the intensity of bands is describes as $v s, s, m, w, v w, s h, b$ and denotes very strong, strong, medium, weak, very weak shoulder and broad, respectively. 
Table S6. The obtained parameters for the DSC measurement of the studied $\mathrm{Cu}$ doped DMAZncompounds. ${ }^{\mathbf{a}}$

\begin{tabular}{|c|c|c|c|}
\hline $\begin{array}{c}\mathrm{Cu} \\
\text { concentration } \\
(\%)\end{array}$ & $\mathbf{T}_{\mathrm{c}}(\mathbf{K})$ & $\begin{array}{c}\Delta S(J \\
\left.\mathrm{mol}^{-1} \mathbf{K}^{-1}\right)\end{array}$ & $\begin{array}{c}\mathbf{N} \text { by } \\
\Delta S=R \ln N\end{array}$ \\
\hline 0 & $\begin{array}{c}167^{\mathrm{u}}, 156^{\mathrm{d}} \\
161.5^{\mathrm{av}}\end{array}$ & $\begin{array}{c}4.25^{\mathrm{u}}, 4.25^{\mathrm{d}} \\
4.25^{\mathrm{av}}\end{array}$ & 1.67 \\
\hline 0.1 & $\begin{array}{c}167^{\mathrm{u}}, 155^{\mathrm{d}} \\
161^{\mathrm{av}}\end{array}$ & $\begin{array}{c}4.15^{\mathrm{u}}, 4.17^{\mathrm{d}} \\
4.16^{\mathrm{av}}\end{array}$ & 1.65 \\
\hline 0.5 & $\begin{array}{c}163^{\mathrm{u}}, 152^{\mathrm{d}} \\
157.5^{\mathrm{av}}\end{array}$ & $\begin{array}{c}4.02^{\mathrm{u}}, 3.96^{\mathrm{d}} \\
3.99^{\mathrm{av}}\end{array}$ & 1.62 \\
\hline 1 & $\begin{array}{c}164^{\mathrm{u}}, 151^{\mathrm{d}} \\
157.5^{\mathrm{av}}\end{array}$ & $\begin{array}{c}3.45^{\mathrm{u}}, 3.47^{\mathrm{d}} \\
3.46^{\mathrm{av}}\end{array}$ & 1.52 \\
\hline 2 & $\begin{array}{c}160^{\mathrm{u}}, 148^{\mathrm{d}} \\
154^{\mathrm{av}}\end{array}$ & $\begin{array}{c}2.46^{\mathrm{u}}, 2.06^{\mathrm{d}} \\
2.26^{\mathrm{av}}\end{array}$ & 1.31 \\
\hline 3 & $\begin{array}{c}156^{\mathrm{u}}, 146^{\mathrm{d}} \\
151^{\mathrm{av}}\end{array}$ & $\begin{array}{c}1.98^{\mathrm{u}}, 1.48^{\mathrm{d}} \\
1.78^{\mathrm{av}}\end{array}$ & 1.24 \\
\hline 5 & $\begin{array}{c}152^{\mathrm{u}}, 136^{\mathrm{d}} \\
144^{\mathrm{av}}\end{array}$ & $\begin{array}{c}1.69^{\mathrm{u}}, 1.20^{\mathrm{d}} \\
1.46^{\mathrm{av}}\end{array}$ & 1.19 \\
\hline
\end{tabular}

aSuperscripts u, d, and av denote heating, cooling, and average, respectively.

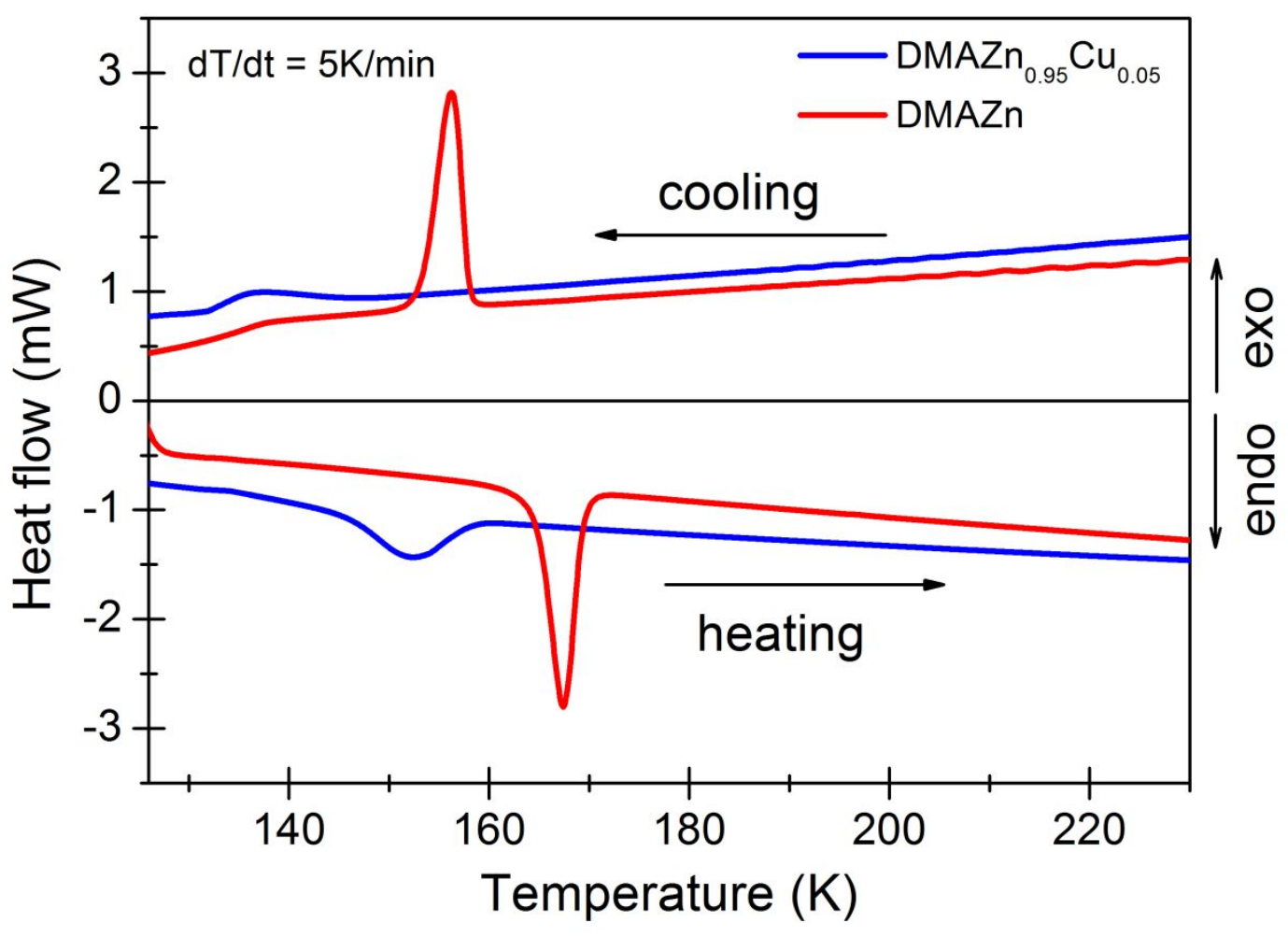

Figure S3. DSC traces for pure DMAZn and $\mathrm{Cu}$ doped DMAZn $\mathrm{n}_{0.95} \mathrm{Cu}_{0.05}$ samples in heating and cooling modes at sweeping rate of $5 \mathrm{~K} / \mathrm{min}$. 
Table S7Lattice parameters for DMAZn from the single-crystal x-ray diffraction.

\begin{tabular}{|l|l|l|l|l|l|l|}
\hline \multicolumn{7}{|c|}{ DMAZn } \\
\hline $\mathbf{T}(\mathbf{K})$ & $\mathbf{a}$ & $\mathbf{b}$ & $\mathbf{c}$ & $\boldsymbol{\alpha}$ & $\boldsymbol{\beta}$ & $\boldsymbol{\gamma}$ \\
\hline 260 & $8.2130(16)$ & $8.2130(16)$ & $22.276(4)$ & 90 & 90 & 120 \\
\hline 240 & $8.2124(16)$ & $8.2124(16)$ & $22.258(5)$ & 90 & 90 & 120 \\
\hline 230 & $8.2125(18)$ & $8.2125(18)$ & $22.253(5)$ & 90 & 90 & 120 \\
\hline 220 & $8.2119(17)$ & $8.2119(17)$ & $22.243(6)$ & 90 & 90 & 120 \\
\hline 210 & $8.2109(16)$ & $8.2109(16)$ & $22.223(5)$ & 90 & 90 & 120 \\
\hline 200 & $8.2101(15)$ & $8.2101(15)$ & $22.212(4)$ & 90 & 90 & 120 \\
\hline 190 & $8.2079(17)$ & $8.2079(17)$ & $22.203(5)$ & 90 & 90 & 120 \\
\hline 180 & $8.2069(16)$ & $8.2069(16)$ & $22.189(5)$ & 90 & 90 & 120 \\
\hline 175 & $8.2065(16)$ & $8.2065(16)$ & $22.188(5)$ & 90 & 90 & 120 \\
\hline 170 & $8.2069(16)$ & $8.2069(16)$ & $22.187(5)$ & 90 & 90 & 120 \\
\hline 165 & $8.2073(16)$ & $8.2073(16)$ & $22.186(5)$ & 90 & 90 & 120 \\
\hline 160 & $8.201(4)$ & $8.201(4)$ & $22.336(17)$ & 90 & 90 & 120 \\
\hline 155 & $8.185(4)$ & $8.185(4)$ & $22.38(2)$ & 90 & 90 & 120 \\
\hline 150 & $8.185(4)$ & $8.185(4)$ & $22.38(2)$ & 90 & 90 & 120 \\
\hline 140 & $8.183(4)$ & $8.183(4)$ & $22.37(2)$ & 90 & 90 & 120 \\
\hline 130 & $8.182(4)$ & $8.182(4)$ & $22.36(2)$ & 90 & 90 & 120 \\
\hline 120 & $8.185(4)$ & $8.185(4)$ & $22.35(2)$ & 90 & 90 & 120 \\
\hline 110 & $8.182(4)$ & $8.182(4)$ & $22.36(2)$ & 90 & 90 & 120 \\
\hline
\end{tabular}

Table S8 Lattice parameters for DMAZn $\mathrm{n}_{0.95} \mathrm{Cu}_{0.05}$ from the single-crystal x-ray diffraction.

\begin{tabular}{|l|l|l|l|l|l|l|}
\hline \multicolumn{7}{|c|}{ DMAZn $_{0.95} \mathrm{Cu}_{0.05}$} \\
\hline $\mathbf{T}(\mathbf{K})$ & $\mathbf{a}$ & $\mathbf{b}$ & $\mathbf{c}$ & $\boldsymbol{\alpha}$ & $\boldsymbol{\beta}$ & $\boldsymbol{\gamma}$ \\
\hline 290 & $8.2109(13)$ & $8.2109(13)$ & $22.270(4)$ & 90 & 90 & 120 \\
\hline 260 & $8.2067(14)$ & $8.2067(14)$ & $22.232(4)$ & 90 & 90 & 120 \\
\hline 240 & $8.2053(13)$ & $8.2053(13)$ & $22.215(4)$ & 90 & 90 & 120 \\
\hline 230 & $8.2029(13)$ & $8.2029(13)$ & $22.197(4)$ & 90 & 90 & 120 \\
\hline 220 & $8.2010(12)$ & $8.2010(12)$ & $22.185(4)$ & 90 & 90 & 120 \\
\hline 210 & $8.1987(13)$ & $8.1987(13)$ & $22.171(4)$ & 90 & 90 & 120 \\
\hline 200 & $8.1983(12)$ & $8.1983(12)$ & $22.162(3)$ & 90 & 90 & 120 \\
\hline 190 & $8.1984(12)$ & $8.1984(12)$ & $22.150(3)$ & 90 & 90 & 120 \\
\hline 180 & $8.1964(12)$ & $8.1964(12)$ & $22.136(3)$ & 90 & 90 & 120 \\
\hline 170 & $8.1958(12)$ & $8.1958(12)$ & $22.124(3)$ & 90 & 90 & 120 \\
\hline 165 & $8.1937(11)$ & $8.1937(11)$ & $22.119(3)$ & 90 & 90 & 120 \\
\hline 160 & $8.1932(11)$ & $8.1932(11)$ & $22.113(3)$ & 90 & 90 & 120 \\
\hline 155 & $8.1923(12)$ & $8.1923(12)$ & $22.105(3)$ & 90 & 90 & 120 \\
\hline 150 & $8.1917(11)$ & $8.1917(11)$ & $22.103(3)$ & 90 & 90 & 120 \\
\hline 140 & $8.1910(12)$ & $8.1910(12)$ & $22.084(4)$ & 90 & 90 & 120 \\
\hline 130 & $8.1883(13)$ & $8.1883(13)$ & $22.075(4)$ & 90 & 90 & 120 \\
\hline 120 & $8.1837(16)$ & $8.1837(16)$ & $22.068(4)$ & 90 & 90 & 120 \\
\hline 110 & $8.1823(17)$ & $8.1823(17)$ & $22.062(4)$ & 90 & 90 & 120 \\
\hline
\end{tabular}




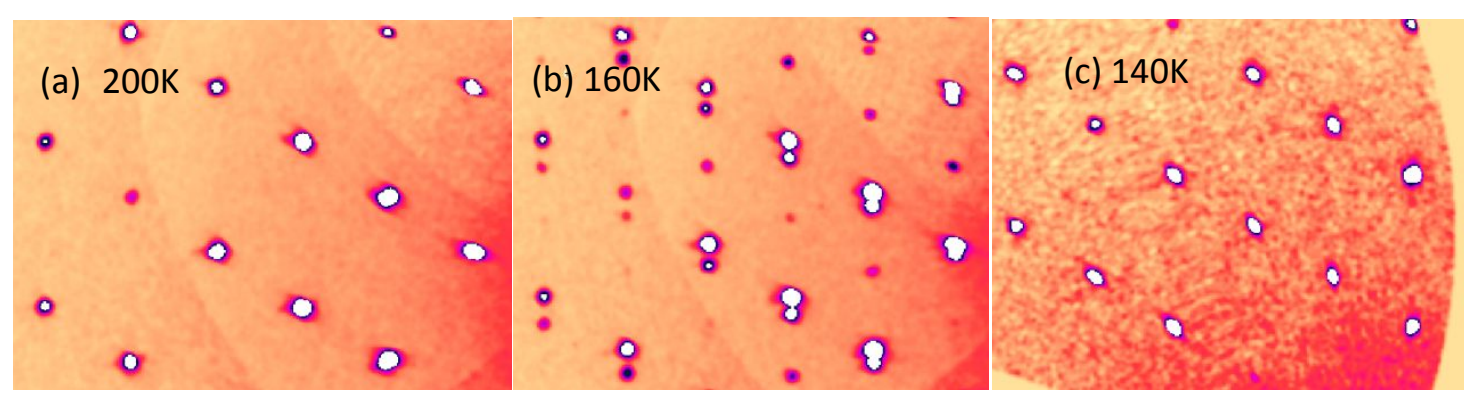

Figure S4 (a) The reconstruction of the reciprocal space for DMAZn (h0l) lattice (in hexagonal setting) at $200 \mathrm{~K}$; (b) at 160K; there is a clear splitting of the diffraction peaks; (c) the reconstruction of the reciprocal space in $\mathrm{DMAZn}_{0.95} \mathrm{Cu}_{0.05}(\mathrm{hOl})$ at $140 \mathrm{~K}$; the splitting is not observed at this temperature. 

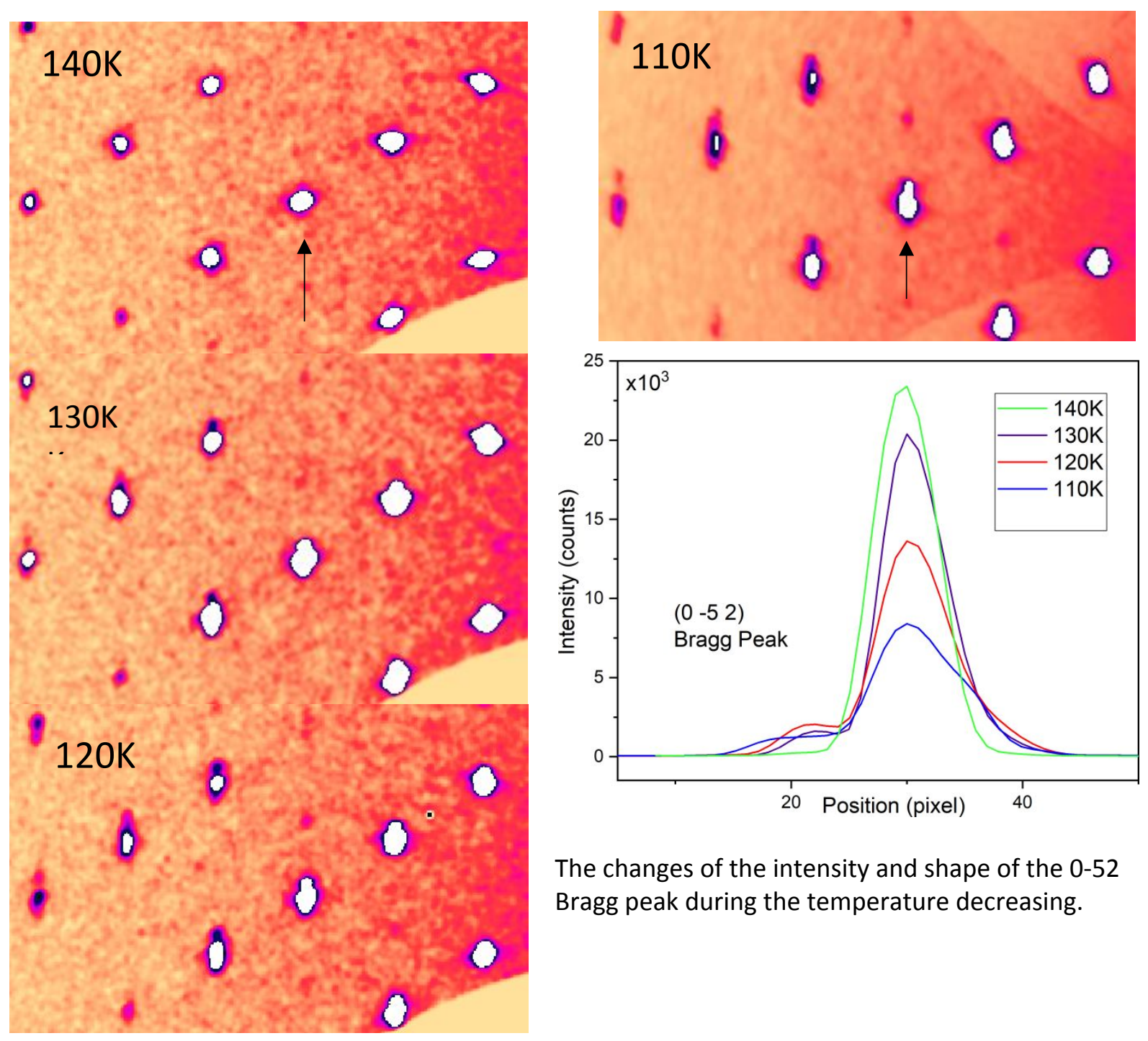

The changes of the intensity and shape of the 0-52 Bragg peak during the temperature decreasing.

Figure S5 The reciprocal space reconstructions of $\mathbf{0 k l}$ layer for DMAZn $\mathbf{n}_{0.95} \mathrm{Cu}_{0.05}$ and the pixel plot of the intensity of 0-52 Bragg peak (marked by arrows)at selected temperatures. 